\title{
Trends and regional variation in the incidence of head and neck cancers in England: 2002 to 2011
}

\author{
CAROLINE E. McCARTHY ${ }^{1,2}$, JOHN K. FIELD ${ }^{2}$, BIJAYA P. RAJLAWAT ${ }^{1}$, \\ ANNE E. FIELD ${ }^{1}$ and MICHAEL W. MARCUS ${ }^{2}$
}

\begin{abstract}
${ }^{1}$ Department of Oral Medicine, Liverpool University Dental Hospital, Royal Liverpool and Broadgreen University Hospitals NHS Trust, Liverpool L7 8XP; ${ }^{2}$ Roy Castle Lung Cancer Research Programme, Department of Molecular and Clinical Cancer Medicine, Institute of Translational Medicine, University of Liverpool, Liverpool L3 9TA, UK
\end{abstract}

Received February 5, 2015; Accepted March 20, 2015

DOI: 10.3892/ijo.2015.2990

\begin{abstract}
Recent studies show an increased incidence of head and neck cancers worldwide. The present study evaluated the trend in the incidence of head and neck cancers in England during 2002-2011. Data were extracted from the database of Office for National Statistics. The study population was categorised according to age, residential area, gender and cancer sub-types. Overall trend in incidence of head and neck cancer and some subtypes were examined using Poisson regression models. In total, 71,457 head and neck cancers were registered in England between 2002 and 2011 and 68\% of patients were males. Statistically significant increases in incidence of 27.0 and $32.4 \%$ were documented in males and females, respectively $(\mathrm{p}<0.001)$ with the largest increase in the $60+$ age category. Potentially HPV-associated cancers, oral cavity cancers and laryngeal cancers increased by $47.1,24.1$ and $1.7 \%$ in males and $37.5,25.5$ and $7.7 \%$ in females, respectively $(\mathrm{p}<0.001)$. Regional differences were also noted with the highest incidence (18.0 and 17.0 per 100,000, respectively) in the North East and North West of England. Our results for England showed an increase in the incidence of both oral cavity and oropharyngeal cancer in both genders, whilst laryngeal cancer incidence remained stable.
\end{abstract}

\section{Introduction}

Head and neck cancer (HNC) includes malignant tumours originating from various sites of the upper aerodigestive tract, the paranasal sinuses and the salivary glands (1). It is the fifth most common cancer worldwide, with over 500,000 cases reported in 2008, with 6076 of these being from the UK (2).

Correspondence to: Dr Michael W. Marcus, Roy Castle Lung Cancer Research Programme, The University of Liverpool Cancer Research Centre, Department of Molecular and Clinical Cancer Medicine, Institute of Translational Medicine, The University of Liverpool, 200 London Road, Liverpool L3 9TA, UK

E-mail: m.w.marcus@liv.ac.uk

Key words: trends, incident, head and neck cancer, England
The incidence of laryngeal cancers has remained stable in recent years, whilst dramatic increases in incidence of oropharyngeal and tonsillar cancers have been reported (3). The major risk factors of HNC are alcohol and tobacco smoking. Other risk factors implicated in the aetiology of HNC include obesity, betel quid chewing, preserved or salted food, low socio economic grouping, gastroesophageal reflux and viruses such as human papilloma virus (HPV), herpes simplex virus (HSV) and Epstein-Barr virus (EBV) (1).

Cancer statistics form part of the evidence-based quality measure to inform decisions regarding public health measures and resource planning. They can also help to define the need for further research in particular areas. In England, cancer registrations are validated by the Office for National Statistics (ONS) following submission of data by the Regional cancer registries. Regional differences in cancer incidence in England have been reported, with the North of England having higher incidence in both males and females, which may be linked to increased levels of deprivation (4). By 2023, it is estimated that $14.7 \%$ of the population will be aged over 70 years, compared to $11.5 \%$ in 2003 . The incidence of cancer is expected to increase in the ageing population and the analysis of trends in incidence will form an important part of health care planning.

The aim of our study was: i) to assess the overall incidence of HNC in England during 2002-2011, ii) to describe the distribution and trends in incidence of HNCs at specific anatomical sites and in the regions of England and iii) to study the trends in incidence between various age-categories and gender.

\section{Materials and methods}

Cases of HNC were identified from the Office for National Statistics (ONS) database, which collects and publishes statistics related to the economy, population and society of the United Kingdom (UK). Cancer registration has been conducted in parts of the UK since 1929 but national coverage was only achieved since 1962. Since then, population-based data on incidence, mortality and survival have been collected by a network of cancer registries across the UK. In England, eight regional cancer registries and London upload their data to a data repository which is then validated by the ONS. The validation processes includes the compatibility of the cancer 
site and the associated histology and are closely based on those promoted by the International Agency for Research on Cancer (5). After validation, detailed results of annual incidences and rates of all types of cancers are published by age, gender and region of residence.

Identification and classification of HNCs. There has been wide variety in the definition of HNC in the literature, with some papers including cervical oesophagus or thyroid gland (6,7). However, our classification was based on the United Kingdom association of cancer registries (UKACR) (8) using International Classification of Diseases, 10th edition (ICD-10). HNC cases (C00-C14 and C30-C32); oral cavity (C00-06, C12-14); salivary gland; (C07-08); tonsil and oropharynx (C09-10); nasopharynx (C11); nasal cavity and middle ear (C30); accessory sinuses (C31); and larynx (C32).

Statistical analyses. Incidence was calculated using cancer registration data and gender- and age-specific population data for each region of England. A ten-year period was chosen in order to have sufficient data to allow examination in recent trends in the incidence of HNC. There has been a reported increase in the incidence of human papilloma virus-related HNC in recent years (9), therefore oropharyngeal (C10), base of tongue (C01) and tonsil cancers (C09) were analysed as a subgroup, as these sites have most frequently been associated with HPV-infection. This group will be referred to as 'Potentially HPV-associated cancers' (HPV-A); The HPV status of these cancers was not known but it has been found that $36-80 \%$ of cancers at these sites are HPV-associated (9).

Oral cavity cancer included ICD 10 codes C00, C02-06 and C12-14, according to the UKACR definition. Laryngeal cancers were also analysed separately (C32). Salivary gland (C07-08), nasopharynx (C11), nasal cavity and middle ear (C30) and accessory sinuses (C31) were not analysed as subgroups but are included in the overall HNC figures. Incident rates were reported as number of new cases per 100,000 personyears, and they were age-standardised according to the 2013 European Standard Population. The study population was categorised according to age, region of residence, gender and cancer sub-types. For this analysis, all HNC patients were divided into five age groups, 0-29, 30-39, 40-49, 50-59, and $60+$ years. Poisson regression models were used to examine time trends in the overall incidence of HNCs and time trends in five age categories, region of residence, gender and HNC specific subtypes in England between 2002 and 2011. All analyses were performed using Stata 13.1 (StataCorp. 2013. Stata Statistical Software: Release 13. College Station, TX: StataCorp LP, USA) and P-values $<0.05$ were considered statistically significant.

\section{Results}

In the period 2002-2011, 71,457 cases of HNC were diagnosed in England: 12,849 were potentially HPV-A, 30,651 were oral cavity cancer and 17,496 were laryngeal cancer. Over two-thirds $(69 \%)$ of HNC were diagnosed in males and $62.8 \%$ of those diagnosed with HNC were 60 years and older (Table I).
Fig. 1 depicts the trend in incidence rates of HNC and the subtypes analysed, for males and females between 2002 and 2011. The incidence rate of HNC increased significantly across all age groups during the study period from 12.2 per 100,000 in 2002 to 15.9 per 100,000 in 2011, a $30.3 \%$ increase $(\mathrm{P}<0.0001)$. When examined by gender, the incidence of HNCs increased by $27.0 \%$ in males (from 17.4 per 100,000 in 2002 to 22.1 per 100,000 in $2011 ; \mathrm{P}=0.003$ ) and by $32.4 \%$ in females ( 7.4 per 100,000 to 9.8 per 100,$000 ; \mathrm{P}=0.004)$. The incidence of potentially HPV-A cancers increased by $45.5 \%$ from 1.8 per 100,000 in 2002 to 3.3 per 100,000 in 2011 $(\mathrm{P}<0.0001)$. Among males, the incidence rate of potentially HPV-A cancers increased by $47.1 \%$ from 2.7 per 100,000 in 2002 to 5.1 per 100,000 in $2011(\mathrm{P}=0.003)$ and among females, the incidence rate increased by $37.5 \%$ from 1.0 per 100,000 to 1.6 per $100,000(\mathrm{P}=0.003)$.

For oral cavity cancer, the incidence increased by $24.6 \%$ from 5.2 per 100,000 in 2002 to 6.9 per 100,000 in 2011 $(\mathrm{P}<0.0001)$. Among males, the incidence increased by $24.1 \%$ from 6.6 per 100,000 in 2002 to 8.7 per 100,000 in 2011 $(\mathrm{P}=0.005)$ and in females by $25.5 \%$ from 3.8 per 100,000 in 20002 to 5.1 per $100,000(\mathrm{P}=0.004)$ in 2011 . In contrast to potentially HPV-A and oral cavity cancers, the incidence of laryngeal cancers was stable. In 2002, the incidence was 3.4 and it increased by $2.9 \%$ to 3.5 per 100,000 in $2011(\mathrm{P}=0.32)$. In males, the incidence increased by $1.7 \%$ from 5.7 in 2002 to 5.8 per 100,000 in $2011(\mathrm{P}=0.4)$ and in females, the incidence increased by $7.7 \%$ from 1.2 in 2002 and 1.3 per 100,000 in $2011(\mathrm{P}=0.40)$.

Regional variation. The incidence of HNC increased significantly in all regions. The North of England has the highest incidence per 100,000. Fig. 2a depicts the trend in incidence of HNC for all regions of England. The North East of England has the highest incidence of HNC in England; in 2011 the incidence was 27.6 and 10.2 per 100,000 for males and females, respectively, compared to the lowest incidence in London of 17.3 per 100,000 males and 6.8 per 100,000 females. The North East also recorded the highest incidence for the subtypes analysed, potentially HPV-A (Fig. 2b), oral cavity cancer (Fig. 2c) and laryngeal cancer (Fig. 2d).

The North West consistently recorded the second highest incidence of HNC from 2002-2011 (average 24.1 per 100,000 males and 9.9 per 100,000 females). The highest number of cases of HNC in England are recorded in the North West; an average of 813 male and 348 female cases of HNC were recorded each year from 2002-2011, compared to 325 and 132, respectively in the North East.

From 2002-2011, there was a significant increase in the incidence of HNC in all regions of England. The most consistent increases were found in the South West and Yorkshire and the Humber for males, with an average annual percentage increase of 3.7 and 3.1\%, respectively. The East Midlands and North West reported the most consistent increases for females with average Annual Percentage Change (APC) $8.1 \%$ and $4.3 \%$, respectively).

Potentially HPV-A cancer incidence increased significantly in males and females in all regions except for females in the East and London. The East Midlands and Yorkshire and the Humber display the most consistent increases in incidence, 
Table I. Total numbers and percentages of head and neck cancer, oral cancer, potentially HPV-associated cancer and laryngeal cancer cases by gender and age category.

\begin{tabular}{lcccc}
\hline Characteristics & $\begin{array}{c}\text { All HNCs } \\
\mathrm{n}=71,457 \\
\mathrm{n}(\%)\end{array}$ & $\begin{array}{c}\text { HPV-related cancers } \\
\mathrm{n}=12,849 \\
\mathrm{n}(\%)\end{array}$ & $\begin{array}{c}\text { Oral cancers } \\
\mathrm{n}=30,651 \\
\mathrm{n}(\%)\end{array}$ & $\begin{array}{c}\text { Laryngeal cancers } \\
\mathrm{n}=17,496 \\
\mathrm{n}(\%)\end{array}$ \\
\hline $\begin{array}{l}\text { Gender } \\
\text { Male }\end{array}$ & & & \\
Female & $49,276(69.0)$ & $9,631(75.0)$ & $19,357(62.0)$ & $14,474(83.0)$ \\
Age group & $22,181(31.0)$ & $3,218(25.0)$ & $11,851(38.0)$ & $3,022(17.0)$ \\
$0-29$ & & & & \\
$30-39$ & $849(1.2)$ & $38(0.3)$ & $273(0.9)$ & $142(0.1)$ \\
$40-49$ & $1,767(2.5)$ & $236(1.8)$ & $769(2.5)$ & $958(5.6)$ \\
$50-59$ & $7,017(9.8)$ & $2,067(16.1)$ & $2,830(9.2)$ & $3,613(20.6)$ \\
$60+$ & $16,981(23.7)$ & $4,461(34.7)$ & $7,114(23.2)$ & $12,760(72.9)$ \\
\hline
\end{tabular}
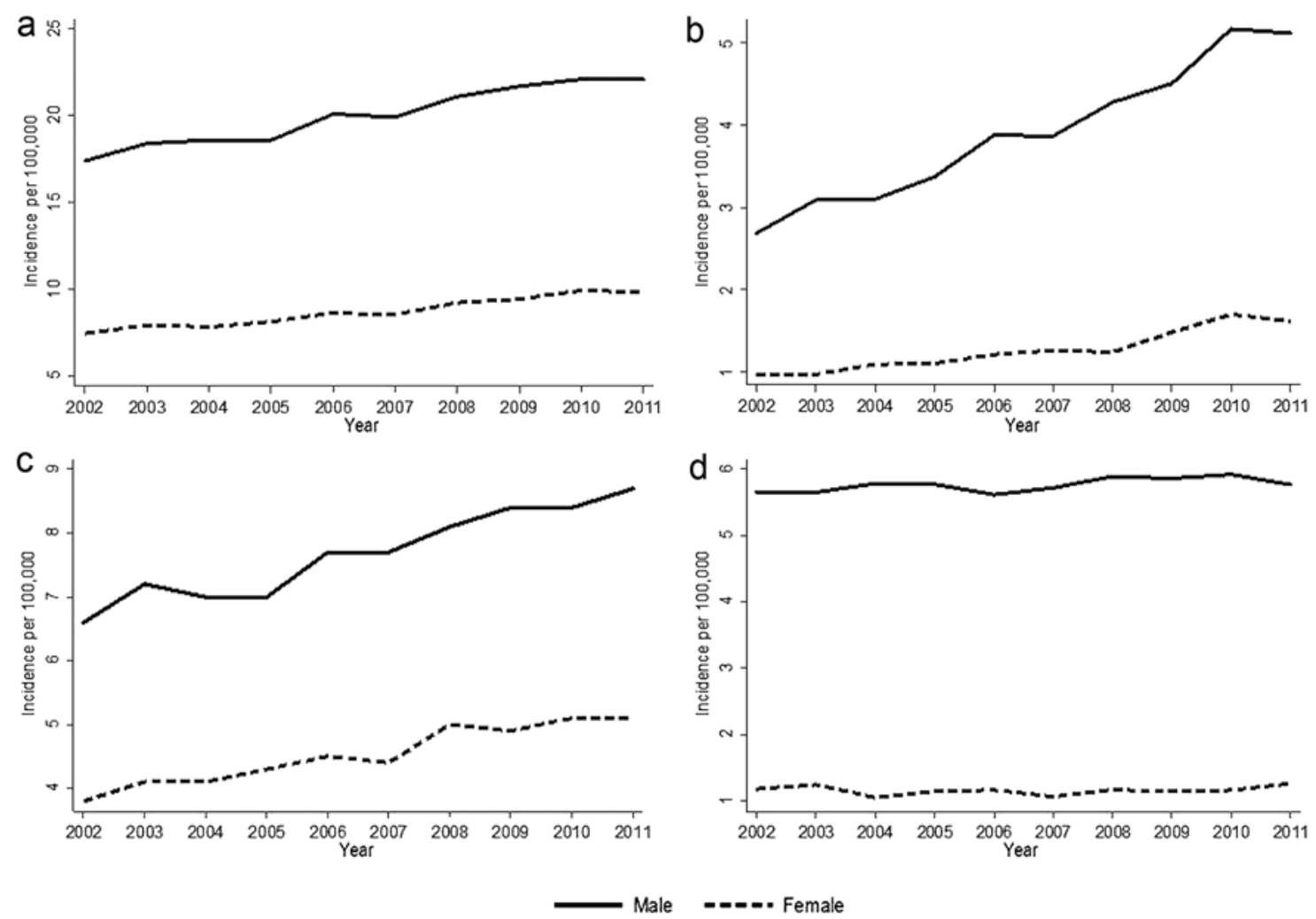

Figure 1. Gender trends in incidence of (a) head and neck cancer, (b) potentially human papilloma virus-associated (HPV-A) cancer, (c) oral cancer and (d) laryngeal cancer in England 2002-2011.

in males, between 2002 and 2011 with average APC of $14.3 \%$ (incidence increased from 1.9 to 5.9 per 100,000) and $8 \%$ (incidence increased from 2.6 to 5.1 per 100,000 ). In females, the most consistent increase was found in the South West, with average APC of $14.1 \%$; incidence increased from 0.9 to 2.1 per 100,000 between 2002 and 2011.

The incidence of oral cavity cancer significantly increased in all regions except Yorkshire and the Humber and the North East, for males and females. There was no significant increase in the East of England for female oral cancers. The
East Midlands and South West displayed the most consistent increases each year for males and females, respectively, with average APC of 5.1\% (incidence increased from 6.2 to 9.2 per 100,000 from 2002-2011) and 5.7\% (incidence increased from 3.5 to 5.6 per 100,000$)$.

The North East showed a statistically significant decrease for Laryngeal cancer in males from 2002-2011 (incidence 9.2 per 100,000 to 7.6 per 100,000). However, the East Midlands report a statistically significant increase in incidence for females, from 0.8 to 1.5 per 100,000 . All other regions display 
a

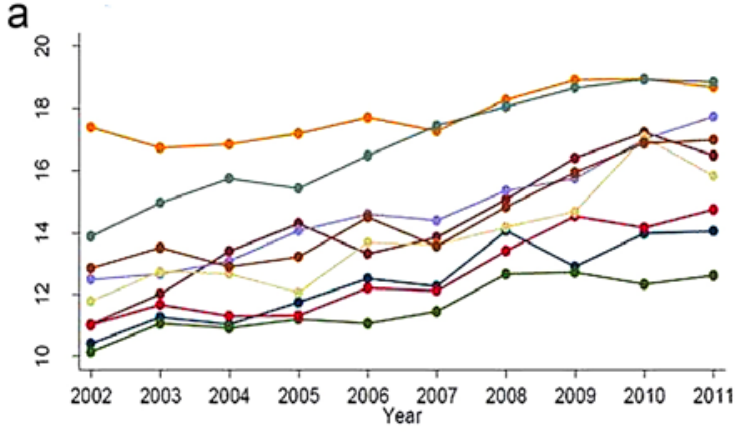

C

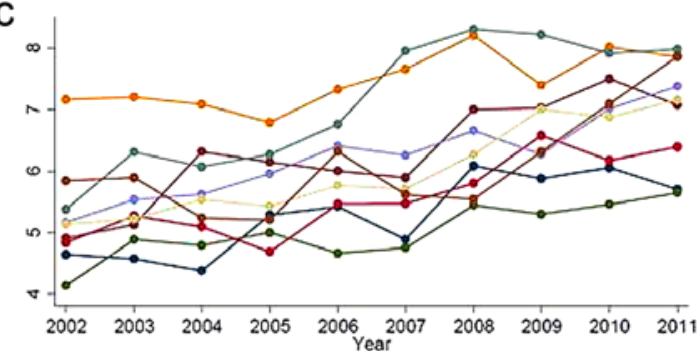

b

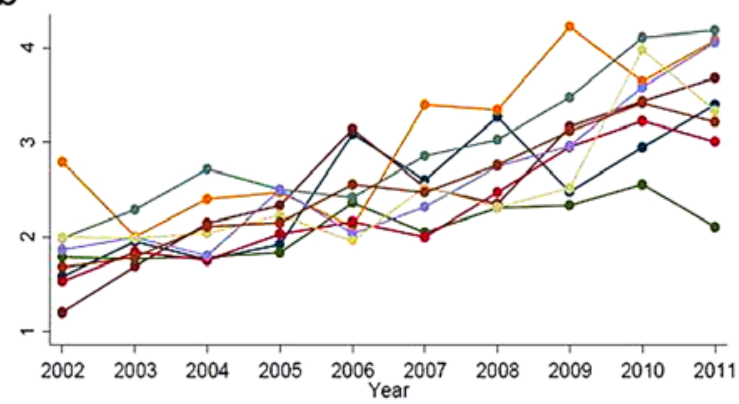

d
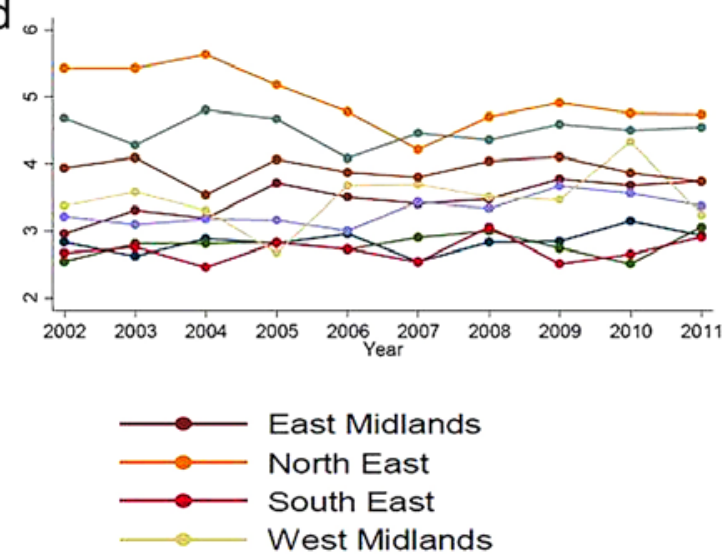

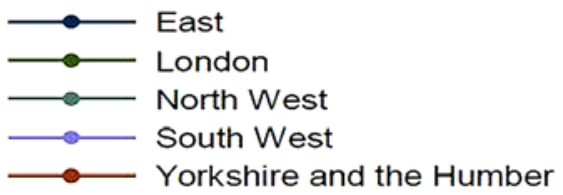

Figure 2. Regional trends in incidence of (a) head and neck cancer, (b) human papilloma virus-associated cancer (HPV-A) cancer, (c) oral cancer and (d) laryngeal cancer in England, 2002-2011.

non-significant trends, indicating the incidence of laryngeal cancer is relatively stable.

\section{Discussion}

This study assessed the trends in incidence of HNC in England in the period 2002-2011, with regards to age, gender and region of residence. More than $96 \%$ of patients were aged above 40 years and $69 \%$ of HNC patients were male, which is similar to figures reported in other studies from England and internationally (10-15).

The incidence of HNC increased significantly during the study period, as did the incidence of cancers at sites known to be highly associated with HPV infection. These findings are also observed by Doobaree et al in their study of HNC in the South East of England between 1995 and 2004 (10). Conway et al observed an increase in oral and pharyngeal cancer rates (C00-C06, C09, C10), in England, between 1990 and 1999, with incidence in males increasing from 6.5 to 8.3 per 100,000 and in females from 2.6 to 3.6 per 100,000 (16). Although our results cannot be directly compared due to differences in classification, the trend appears to be similar over the two decades.

We found no significant increase in the incidence of laryngeal cancer between 2002 and 2011. This finding is also reported by the Oxford Cancer Intelligence Unit in a report detailing the profiles of HNC in England. They found that the incidence of laryngeal cancer reduced by $16.7 \%$, from 3.6 per 100,000 to 3.0 per 100,000 , between 1990 and 2006 but stabilised in the latter five years (3). This is supported by
Coupland et al, who found that incidence has decreased from the early 1990's, particularly in the over 70's age group (17). A reduction in laryngeal cancer incidence in males and stable incidence in females has been reported in France, Finland, Norway, Denmark, Spain and the Netherlands $(2,14,18)$. The highest incidence of laryngeal cancer was found in the $60+$ age category. Subgroup analysis of the this age group showed that the highest incidence of laryngeal cancer was found in 75-84 year olds which is similar to findings reported from the Netherlands that $21 \%$ of patients are in the $75-85$ year age group (18).

Age. More than two-thirds of all HNC patients were in the $60+$ year age group from 2002-2011. Dobaree et al reported that $60 \%$ of HNC patients were aged 40-69 years in South East England between 2000 and 2004. A study conducted in Germany reported a similar age at diagnosis, with $50 \%$ of HNC patients being aged $60-79$ years $(10,11)$ and a study in the United States reported mean age at diagnosis of 62 years (19) (www.seer.cancer.gov/faststats/selections.php). Over the ten year period we studied, the most marked increases in HNC incidence were found in the 55-59 year age category for males and 65-69 year age category for females, which is mostly due to the increase in oropharyngeal, base of tongue and tonsil cancer as discussed below.

The highest incidence of oral cavity cancer was found in the over 80 's, for both males and females. The most significant increases in incidence of oral cavity cancer were found in males aged 60-64 years and females aged 65-69 years. Results from Portugal show that the highest incidence of oral 
cancer in females is in the over 75 year age group, whereas for males, the 60-64 year age group has highest incidence, further supporting the concept that females with oral cancer tend to be older than males (13). Oral cancer incidence is increasing in the Netherlands in females; however, rates are stable in males. This observation is similar to those reported from France $(18,20)$. Other studies have reported reduced incidence of oral cancer; a reduction of $1.5 \%$ per year was found between 1995 and 2004 in the United States (20). However, in the time period 2003-2010, there was an annual increase of $0.2 \%$ in oral cancers in males (19). In fact, cancers of the tongue increased in males by $2.4 \%$ annually between 1999 and 2010 and in females by $0.6 \%$ annually between 1992 and 2010.

Information on HPV-status was not available from the ONS, therefore our classification of potentially HPV-A as cancers of the tonsil, oropharynx and base of tongue is based on reports in the literature that have found between $36 \%$ and $>80 \%$ of cancers at these sites are infected by HPV, implying, but not proving, causation (9). A recent study confirmed 55\% of oropharyngeal cancers were HPV-infected and HPV infection poses an increased risk of developing oropharyngeal cancer: OR 3.5 (95\% CI 2.1-5.9) $(21,22)$.

In our study, potentially HPV-A cancer seems to affect younger individuals, with the highest incidence found in those aged 60 years and above; it also affects proportionally more people aged 40-49 years than other HNC. Other $\mathrm{HNC}$ have higher incidence in older age categories, however, incidence of potentially HPV-A cancer decreases in persons aged over 65 years. The incidence of potentially HPV-A cancer doubled in males aged 55-59 and 65-69 years and almost doubled in females aged 65-69 years between 2002 and 2011. This finding is in agreement with National Cancer Information Service data, which show that the incidence of oropharyngeal cancer doubled in England between 1990 and 2006 (3); this rise is thought to be due to HPV-infection. However, the incidence increased sharply between 2006 and 2011 perhaps also because of increased HPV-infection (Fig. 1b).

Researchers from Canada have also reported a marked increase in tonsil and base of tongue cancer, again, believed to be due to HPV infection (23). They reported the largest increase in persons aged 50-59 years (incidence 4.4 to 8.9 per 100,000 between 1997 and 2009), which is similar to our findings in England. Researchers from Portugal reported that oropharyngeal cancer in men increased by $3.5 \%$ per year and in females by 2\% per year between 1998 and 2007 (13) and researchers from the United States reported 2.3\% annual increase in the incidence of oropharyngeal and tonsillar cancer for males between 1992 and 2010 (19,24). Of note, a 0.4\% annual reduction in female oropharyngeal and tonsil cancers is reported in the United States in the period 1992-2010 (19) but the reason for this increase is unclear.

Gender. The ratio of males: females HNC remained relatively stable over the study period (approximately 2:1). The incidence of HNC in males is higher than females throughout all age groups; however, the relative proportion of females affected increases in the older age categories, likely due to a larger female population. The male: female ratio is most even for cancer of the oral cavity; subgroup analysis showed that there are more female oral cavity cancer patients over 80 years of age than male patients (58 and $42 \%$, respectively). Females have longer life-expectancy than males and the population of females is much larger in this age group $(1,564,400$ and 920,700 , respectively in 2011).

Laryngeal cancer had the highest ratio of male:female cancers (4.6:1) and this ratio is very similar throughout all age groups, even the most elderly, which is different to the trend observed with oral cavity cancer: Coupland $e t$ al reports a male:female ratio of 4.8:1 for laryngeal cancer in the South East of England between 1985 and 2004 (17). In other countries, the ratio is much higher: Lithuania and Portugal have a male:female ratio of $25: 1$ and $36: 1$, respectively (2). The incidence in males varies widely from country to country from 11.9 per 100,000 in Hungary to 1.8 per 100,000 in Sweden (2). Smoking and alcohol consumption are the major risk factors for all HNC, however, for laryngeal cancer the Population Attributable Risk (PAR) has been found to be $89 \%$ compared to $64 \%$ for oral cavity cancer (25). Males also have a higher PAR for smoking and alcohol compared to women (74\% compared to $57 \%$, respectively) (25); these findings may help to explain the much higher incidence in males than females.

Region. The North East and North West have the highest incidence of $\mathrm{HNC}$ and all subtypes analysed, with incidence rates that are consistently above the national average. For HNC overall (C00-14, C30-32), all Regions of England showed a significant increase in incidence in the period 2002-2011. Not all regions had a statistically significant increase in oral cavity cancer but none displayed a decrease in incidence. Incidence of potentially HPV-A cancers increased in all regions for males, particularly the East Midlands and Yorkshire and the Humber; interestingly Yorkshire and the Humber was one of the regions with no statistically significant increase in oral cancer incidence. For females, it was the South West with the most marked increase in potentially HPV-A cancers. Laryngeal cancer incidence significantly decreased in the North East, although even with the decrease, the incidence is still the highest in the country. A previous study has found a decreasing incidence in laryngeal cancer from the North to the South of England, thought to be due to the changes in the industrial landscape and supporting the concept of the 'North-South divide', which is a term used to describe gross differences in socio-economic status for individuals living in the North and South of England (3).

It has previously been reported that there is increased incidence of HNC in lower socio-economic groups and a link with deprivation has been established (26). Since smoking and alcohol consumption are the two most significant risk factors for $\mathrm{HNC}$, differences in the rates of smoking and alcohol consumption could help to explain the regional variations in incidence of the disease. Smoking rates in the UK reduced dramatically between 1974 and 2011 (51-20\% of men and 41-18\% of women) but regional variations still exist: in 2011 smoking rates were higher in the North West (22\%) than the rest of the England (20\%), although this is not statistically significant (27) Residential area deprivation is a strong independent predictor for smoking (OR 1.85 CI 1.57-2.13) and the North West contains over half of the $1 \%$ most deprived areas of 
England (28,29). Smoking is more prevalent in the routine and manual occupations than managerial and professional (29 vs. $14 \%$ for males; 26 vs. $12 \%$ for females) and the percentage of never smokers is lower. It is known that people in lower socio-economic groups are not only more likely to smoke but generally start smoking at younger age, smoke more heavily and are less likely to quit smoking, each of which increases their risk of HNC (27).

Heavy drinking on at least one day in the week ( $>8$ units for men, $>6$ units for women) was most common in the North West and Yorkshire and the Humber in 2011 (23 vs. 18\%). In contrast to smoking, regular alcohol consumption (at least 5 days per week) is more prevalent amongst the managerial/ professional group than the routine/manual group (19 vs. $13 \%$ for males) and in those who are in employment compared to the unemployed (15 vs. $6 \%$ for males) (27). Smoking and drinking are also closely linked: according to ONS data (2011), 14\% of male smokers consumed greater than 12 units of alcohol on one day in the preceding week compared to $8 \%$ of nonsmokers. It has also been found that, amongst young people aged 11-15 years, occasional and regular smokers are much more likely to drink alcohol (OR 2.85 and 3.65, respectively) than non-smokers (30).

Ethnicity has also been considered as a risk factor for $\mathrm{HNC}$, due to the marked differences in incidence of HNC around the world. However, some of the differences are cultural rather than ethnic e.g. chewing betel or tobacco consumption. These may not be transferred along ethnic lines due to availability in England. Incidence of HNC is highest in South and South East Asia and the incidence of HNC amongst South East Asians living in the UK is higher than in other ethnic groups $(6,31)$ : Csikar et al reported an incidence of 7.2 per 100,000 and 6.0 per 100,000, respectively. They concluded that areas in which many South Asian women live, there may be a higher incidence of head and neck (particularly oral) cancer. However, London has the highest percentage of Indian, Bangladeshi and Pakistani persons in England (1.8\% of the population) and our study shows the incidence of HNC was the lowest of all the Regions (16.4 per 100,000 males and 6.8 per 100,000 females). Smokeless tobacco use is highest in Bangladeshi women (16\% of the Bangladeshi population) and this is thought to account for the higher incidence of oral cancer in this group (6). Noteworthy, there appears to be no link between oral and pharyngeal cancer with deprivation, in South Asian males and females living in the UK. Presumably, we cannot compare deprivation in South Asia (poor sanitation and poor diet) with deprivation in the UK - smoking, drinking and poor diet. This supports the concept that ethnic-specific risk factors account for the higher incidence of oral/HNC in this group of the population (31).

In conclusion, our study has confirmed that the incidence of head and neck cancer continues to rise in England. Between 2002 and 2011, incidence increased from 12.2 to 15.9 per 100,000 . Oral cavity cancer incidence is also increasing, in males and females, whereas incidence of laryngeal cancer is stable. The incidence of cancers at sites strongly associated with HPV infection doubled, in males and females in high-risk age-groups in this ten-year period. Regional variation exists and further work is needed to establish the role of deprivation and socioeconomic status on HNC incidence. Cancer statis- tics form an important part of health care planning and this information may be used to inform researchers when planning studies, screening and health awareness programmes in different Regions of England.

\section{Acknowledgements}

Dr Michael Marcus was supported by grants from the European Community's Seventh Framework Programme (FP7/20072013) under grant agreement no. HEALTH-F2-2010-258677 (CURELUNG project) and grant agreement no. 258868 (Lung Cancer Artificial Olfactory System Project).

\section{References}

1. Marcus MW, Chen Y, Liloglou T and Field JK: New perspectives to respiratory tract cancers. Head Neck Oncol 5: 28, 2013.

2. Ferlay JSH, Bray F, Forman D, Mathers C and Parkin DM: GLOBOCAN 2008 v2.0, Cancer Incidence and Mortality Worldwide: IARC CancerBase No. 10 [Internet]. Lyon, International Agency for Research on Cancer. Available from: http://globocan.iarc.fr. Accessed on March 11, 2013.

3. Price G, Roche M, Crowther R and Wright R: Profiles of head and neck cancers in England: Incidence. Mortality and Survival 120: 1990-1997, 2010

4. Quinn M BP, Brock A, Kirby L and Jones J: Office for National Statistics: Cancer Trends in England and Wales, 1950-1999, SMPS No. 66. 2005.

5. Parkin DM, Chen VW, Ferlay J, Galceran J, Storm HH and Whelan SL: Comparability and quality control in cancer registration. IARC Technical Report No. 19, Lyon, 1994.

6. Csikar J, Aravani A, Godson J, Day M and Wilkinson J: Incidence of oral cancer among South Asians and those of other ethnic groups by sex in West Yorkshire and England, 2001-2006. Br J Oral Maxillofac Surg 51: 25-29, 2013.

7. Davies L and Welch HG: Epidemiology of head and neck cancer in the United States. Otolaryngol Head Neck Surg 135: 451-457, 2006.

8. UKACR UKaocr: Library of Recommendations on Coding and Classification Policy and Practice agreed by the UKACR Executive Committee. p56, 2002.

9. Kreimer AR, Clifford GM, Boyle P and Franceschi S: Human papillomavirus types in head and neck squamous cell carcinomas worldwide: A systematic review. Cancer Epidemiol Biomarkers Prev 14: 467-475, 2005.

10. Doobaree IU, Landis SH, Linklater KM, El-Hariry I, Moller H and Tyczynski J: Head and neck cancer in South East England between 1995-1999 and 2000-2004: An estimation of incidence and distribution by site, stage and histological type. Oral Oncol 45: 809-814, 2009 .

11. Hertrampf K, Wiltfang J, Katalinic A, Timm O and Wenz HJ: Trends in incidence, tumour sites and tumour stages of oral and pharyngeal cancer in Northern Germany. J Cancer Res Clin Oncol 138: 431-437, 2012.

12. Yeole BB: Trends in incidence of head and neck cancers in India. Asian Pac J Cancer Prev 8: 607-612, 2007.

13. Monteiro LS, Antunes L, Bento MJ and Warnakulasuriya S: Incidence rates and trends of lip, oral and oro-pharyngeal cancers in Portugal. J Oral Pathol Med 42: 345-351, 2013.

14. Blomberg M, Nielsen A, Munk C and Kjaer SK: Trends in head and neck cancer incidence in Denmark, 1978-2007: Focus on human papillomavirus associated sites. Int J Cancer 129: 733-741, 2011.

15. Attar E, Dey S, Hablas A, Seifeldin IA, Ramadan M, Rozek LS and Soliman AS: Head and neck cancer in a developing country: A population-based perspective across 8 years. Oral Oncol 46: 591-596, 2010.

16. Conway DI, Stockton DL, Warnakulasuriya KA, Ogden G and Macpherson LM: Incidence of oral and oropharyngeal cancer in United Kingdom (1990-1999) - recent trends and regional variation. Oral Oncol 42: 586-592, 2006.

17. Coupland VH, Chapman P, Linklater KM, Sehgal A, Møller H and Davies EA: Trends in the epidemiology of larynx and lung cancer in south-east England, 1985-2004. Br J Cancer 100: 167-169, 2009. 
18. Braakhuis BJ, Visser O and Leemans CR: Oral and oropharyngeal cancer in The Netherlands between 1989 and 2006: Increasing incidence, but not in young adults. Oral Oncol 45: e85-e89, 2009

19. National Cancer Institute: Surveillance, Epidemiology and End Results Program. www.seer.cancer.gov/faststats/selections.php.

20. Warnakulasuriya S: Global epidemiology of oral and oropharyngeal cancer. Oral Oncol 45: 309-316, 2009.

21. Evans M, Newcombe R, Fiander A, Powell J, Rolles M, Thavaraj S, Robinson M and Powell N: Human Papillomavirusassociated oropharyngeal cancer: An observational study of diagnosis, prevalence and prognosis in a UK population. BMC Cancer 13: 220, 2013.

22. Herrero R, Castellsagué X, Pawlita M, Lissowska J, Kee F, Balaram P, Rajkumar T, Sridhar H, Rose B, Pintos J, et al; IARC Multicenter Oral Cancer Study Group: Human papillomavirus and oral cancer: The International Agency for Research on Cancer multicenter study. J Natl Cancer Inst 95: 1772-1783, 2003.

23. Forte T, Niu J, Lockwood GA and Bryant HE: Incidence trends in head and neck cancers and human papillomavirus (HPV)associated oropharyngeal cancer in Canada, 1992-2009. Cancer Causes Control 23: 1343-1348, 2012.

24. Cooper JS, Porter K, Mallin K, Hoffman HT, Weber RS, Ang KK, Gay EG and Langer CJ: National Cancer Database report on cancer of the head and neck: 10-year update. Head Neck 31: 748-758, 2009.
25. Hashibe M, Brennan P, Chuang SC, Boccia S, Castellsague X, Chen C, Curado MP, Dal Maso L, Daudt AW, Fabianova E, et al: Interaction between tobacco and alcohol use and the risk of head and neck cancer: Pooled analysis in the International Head and Neck Cancer Epidemiology Consortium. Cancer Epidemiol Biomarkers Prev 18: 541-550, 2009.

26. Sharpe KH, McMahon AD, McClements P, Watling C, Brewster DH and Conway DI: Socioeconomic inequalities in incidence of lung and upper aero-digestive tract cancer by age, tumour subtype and sex: A population-based study in Scotland (2000-2007). Cancer Epidemiol 36: e164-e170, 2012.

27. Office for National Statistics: General Lifestyle Survey. 2011.

28. Shohaimi S, Luben R, Wareham N, Day N, Bingham S, Welch A, Oakes $\mathrm{S}$ and Khaw KT: Residential area deprivation predicts smoking habit independently of individual educational level and occupational social class. A cross sectional study in the Norfolk cohort of the European Investigation into Cancer (EPIC-Norfolk). J Epidemiol Community Health 57: 270-276, 2003.

29. Department for Communities and Local Government: English Indices of Deprivation 2010.

30. Health and Social Care Information Centre: Smoking, drinking and drug use among young people in England in 2009. NHS Information Centre for Health and Social Care, 2010.

31. Moles DR, Fedele S, Speight PM, Porter SR and dos Santos Silva I: Oral and pharyngeal cancer in South Asians and non-South Asians in relation to socioeconomic deprivation in South East England. Br J Cancer 98: 633-635, 2008. 